Terr. Atmos. Ocean. Sci., Vol. 18, No. 1, 97-116, March 2007

\title{
Analysis of Terminal Velocity and VHF Backscatter of Precipitation Particles Using Chung-Li VHF Radar Combined with Ground-Based Disdrometer
}

\author{
Ching-Lun $\mathrm{Su}^{1}$, and Yen-Hsyang $\mathrm{Chu}^{1}{ }^{1}$ *
}

(Manuscript received 22 May 2006, in final form 6 November 2006)

\begin{abstract}
The backscatter from precipitation particles observed by the vertically pointed antenna beam of the Chung-Li VHF radar and the drop size distributions measured by a ground-based disdrometer co-located at the radar site are analyzed and studied in this article. We find that the disdrometermeasured drop size distribution can be well approximated to a Gamma distribution. On the basis of this property and a power law approximation to the fallspeed-diameter relation $V_{D}=A D^{B}$, we derive the theoretical relation between terminal velocity $V_{D}$ and range-corrected $V H F$ backscatter $P$ of the precipitation particles. We find that the $V_{D}-P$ relation follows a power law in the form of $V_{D}=\alpha P^{\beta}$, where $\alpha$ and $\beta$ are both the functions of the precipitation parameters. Chu et al. (1999) first found that the relation between $\alpha$ and $\beta$ can be empirically approximated to an exponential form of $\alpha=A \boldsymbol{e}^{\xi \beta}$, where $\beta$ is a function of $\boldsymbol{B}$ and $\xi$ is a factor associated with precipitation. In this article, under the assumptions of the Gamma distribution of the drop size distribution and the power-law relation between $V_{D}$ and $D$, we theoretically show that the analytical relation between $\alpha$ and $\beta$ indeed follows an exponential form of $\alpha=A e^{\xi \beta}$, where $\xi$ is a function of the drop size distribution. The experimental results obtained by the Chung-Li VHF radar combined with the ground-based disdrometer measurements validate the exponential approximation to the $\alpha-\beta$ relation. The uses of the $\alpha-\beta$ relation for the investigations of the rainfall rate and properties of drop size distribution are presented and discussed.
\end{abstract}

(Key words: VHF Radar, Precipitation, Terminal velovity, Reflectivity)

\footnotetext{
${ }^{1}$ Institute of Space Science, National Central University, Chung-Li, Taiwan, ROC

* Corresponding author address: Prof. Yen-Hsyang Chu, Institute of Space Science, National Central University, Chung-Li, Taiwan, ROC; E-mail: yhchu@jupiter.ss.ncu.edu.tw
} 


\section{INTRODUCTION}

Theoretical and experimental results both show that the Doppler radar operating at VHF band (or VHF wind profiler) cannot only measure the echoes from refractive index fluctuations of the clear air, but also effectively detect the backscatter from precipitation particles (Larsen and Rottger 1987; Chu and Chian 1991). This unique capability makes the VHF Doppler radar capable of measuring the true terminal velocity of the precipitation particles and other crucial precipitation-related parameters and features after carefully removing clear air effects from observed precipitation echoes. For example, Wakasugi et al. (1986) developed a method to deduce the size distribution of the precipitation particles aloft from VHF precipitation echoes, in which the beam broadening effect caused by horizontal wind blowing across the radar volume on the observed precipitation Doppler spectral width is removed. Chu and Lin (1994) studied the severe depletion of the VHF clear-air echo intensity associated with intense updraft in the precipitating environment. Rottger et al. (1995) investigated the characteristics of lightning echoes with spatial interferometry technique implemented on the Chung-Li VHF radar. Chu et al. (1997) conducted an experiment with VHF Doppler radar to explore the nonfrozen property of a hydrometeor drifting in the background wind. Rao et al. (1999) utilized the Indian VHF radar to study the tropical precipitation system and bright band structure from precipitation echoes. Reddy et al. (2002) estimated the horizontal drift velocity of the tropical depression-associated rain cell from VHF precipitation echoes with the spaced antenna drift method.

The relations between the mean terminal velocity $V_{D}$ and the radar backscatter $P$ (or radar reflectivity factor $Z$ ) from precipitation particles have been also studied by a number of scientific workers (Chilson et al. 1993; Ulbrich and Chilson 1994; Chu et al. 1999; Su et al. 2004). With VHF/UHF radars, Chilson et al. (1993) analyzed the relationship between $V_{D}$ and $Z$ associated with a thunderstorm and found the relationships of $V_{D}=0.21 \mathrm{Z}^{0.34}$ and $V_{D}=0.91 \mathrm{Z}^{0.16}$, where the former corresponds to the data taken during the early development of the storm and the latter for the convective region. Chu et al. (1999) analyzed precipitation data taken from 4 independent experiments conducted with the Chung-Li VHF radar. They found that $\alpha$ and $\beta$ values in the power-law approximation $V_{D}=\alpha P^{\beta}$ above the melting layer are generally smaller than those below the layer, while in the height range of bright band the values of $\beta(\alpha)$ are enormously smaller (greater) than those above and below the bright band.

In addition to the investigation of $V_{D}-P$ relations, Chu et al. (1999) found that the relation between $\alpha$ and $\beta$ can be empirically approximated very well to a simple exponential function $\alpha=A e^{-\xi \beta}$, where $\mathrm{A}$ is the coefficient in the power-law approximation to the fallspeeddiameter relationship $V_{T}(D)=A D^{B}$ of a precipitation particle with respect to the still air and $\xi$ is a parameter with unknown analytical expression. This empirical relation between $\alpha$ and $\beta$ has been validated by using the Chung-Li VHF radar and found to be in excellent agreement with the radar measurements. On the basis of the work of Chu et al. (1999), Su et al. (2004) theoretically showed that the $\alpha-\beta$ relation does indeed follow an exponential form, validating the empirical expression obtained by Chu et al. (1999).

In this article, an attempt is made to theoretically verify that the mathematical relation between $\alpha$ and $\beta$ follows the exponential form. The applications of the derived $\alpha-\beta$ rela- 
tion to the estimations of rainfall rate and the parameters of the rain drop size distribution are also discussed. In section 2, with the assumptions of Gamma drop size distribution and powerlaw approximation to the $V_{T}-D$ relation, the $V_{D}-P$ relationship for VHF radar returns is theoretically analyzed. In addition, with the help of the asymptotic approximation of Gamma function, the mathematical relation between $\alpha$ and $\beta$ in the power-law relation $V_{D}=\alpha P^{\beta}$ is derived. We find that $\alpha-\beta$ relation is in an exponential form of $\alpha=A e^{\xi \beta}$, where $\xi$ is a function of radar and precipitation parameters. In section 3 , the applicability of an exponential approximation to $\alpha-\beta$ relation is examined. We find that the observational result is in excellent agreement with the theoretical expectation. In section 4 , the primary properties of the $\alpha-\beta$ relation are discussed and its applications to the estimations of the rainfall rate and drop size distribution are also investigated. We show that the $\alpha-\beta$ relation obtained by $\mathrm{Su}$ et al. (2004) is only a special case of the present result. The conclusion is drawn in section 5.

\section{THEORETICAL CONSIDERATIONS}

It is well known that the radar equation for the precipitation echoes observed by a VHF radar can be formulated as below (Battan 1973):

$$
P_{r}=\frac{C|K|^{2} Z}{r^{2}}
$$

where $r$ is the range, $K=\left(m^{2}-1\right) /\left(m^{2}+1\right)$, $\mathrm{m}$ is the complex refractive index of a rain drop, and $|K|^{2}=0.93$ for a rain drop at temperature around $10^{\circ} \mathrm{C}, Z$ is the radar reflectivity factor and is defined as:

$$
Z=\sum D_{i}^{6}=\int N(D) D^{6} d D
$$

where $N(D) d D$ is the number of precipitation particles with diameter within $D$ and $D+d D$ per unit volume, and $C$ in expression (1) is a constant related to radar parameter in accordance with the following expression:

$$
C=\frac{L c P_{t} G G_{t} G_{r} \pi^{3} \tau \theta \psi}{1024 \lambda^{2} \ln (2)}
$$

where $L$ is radar efficiency, $\theta$ is the antenna beam width in major axis, $\psi$ is antenna beam width in minor axis, $P_{t}$ is transmitted peak power, $G_{r}$ and $G_{t}$ are, respectively, receiving and transmitting antenna gains, $c$ is light speed, $\tau$ is pulse width, $\lambda$ is radar wavelength, $G$ is radar transfer gain. It should be noted that the expressions (1) - (3) can only be applicable to the precipitation particles with spherical shape at the diameter much smaller than radar wavelength such that the echoing mechanism of the Rayleigh scattering is valid. In addition, the effects of the range weighting and the rain attenuation on the amplitude of radar returns are 
also not considered in (1) - (3). From (3), we note that the unit of $C$ is $\mathrm{W} \mathrm{m}^{-1}$ if the MKS unit system is adopted. However, conventionally, the unit of $Z$ in (2) is $\mathrm{m}^{-3} \mathrm{~mm}^{6}$ if $D$ is in unit $\mathrm{mm}$ and $N(D)$ is in unit $\mathrm{m}^{-3} \mathrm{~mm}^{-1}$. As a result, the right hand side of expression (1) is required to be multiplied by a factor of $10^{-18}$ such that the units in both sides of (1) are equal. Assume the size distribution $N(D)$ of precipitation particle follows a Gamma distribution and the relation between fallspeed $\mathrm{V}_{\mathrm{T}}$ with respect to still air and the diameter $D$ of a precipitation particle follows the power law, namely:

$$
N(D)=N_{o} D^{\mu} \exp (-\delta D)
$$

and

$$
V_{T}(D)=A D^{B} .
$$

Expression (5) has been employed extensively by researchers for the investigations of drop size distribution, terminal velocity-radar backscatter relation, and other applications (Atlas et al. 1973; Leary and House 1979; Hauser and Amayenc 1981; Caylor and Illingworth 1987; Chu et al. 1999). Theoretical and experimental results both showed that the magnitude of $B$ is in the range 0.5 - 0.7 (Spilhaus 1948; Sekhon and Srivastava 1971; Atlas and Ulbrich 1977; Chu et al. 1999). It is worth pointing out that the expression (5) cannot be applied to a raindrop with a diameter greater than about $6 \mathrm{~mm}$. Beyond that, the drop may become unstable and is likely to be broken up due to vibration and deformation of the drop itself (Gunn and Kinzer 1949).

From (4), we note that the characteristics of the Gamma distribution are determined by three parameters, namely, $N_{o}, \delta$, and $\mu$. It is not difficult to show that the mode $D_{o}$, mean $D_{m}$ and variance $\sigma$ (defined as the second moment with respect to mean) of the Gamma distribution are related to the parameters $N_{o}, \delta$, and $\mu$ in accordance with following expressions, respectively:

$$
\begin{aligned}
& D_{o}=\frac{\mu}{\delta}, \\
& D_{m}=D_{o}+\frac{1}{\delta}, \\
& \sigma^{2}=D_{m}\left(D_{m}-D_{o}\right) .
\end{aligned}
$$

From (6) - (8), it is clear to see that the parameters $\mu$ and $\delta$ not only determine the mode and mean, but also govern the shape and breadth of the Gamma function. From (7), because $D_{m}$ is greater than $D_{o}$ due to $\delta>0$, the skewness (in the sense close to the difference between $D_{m}$ and $D_{o}$ ) of the Gamma distribution will be always positive, namely, skewing toward the smaller end of the drop size. Moreover, the coefficient of variance (defined as the ratio of $\sigma^{2}$ to $D_{m}{ }^{2}$ ) is determined solely by the parameter $\mu$ and the relation $\mu=D_{o} /\left(D_{m}-D_{o}\right)$ can be readily obtained from (6) and (7). Let the drop number density at the size of $D_{o}$ be $N_{D}$. Substituting 
(6) into (4) and replacing $D$ by $D_{o}$, we have:

$$
N_{o}=N_{D} D_{o}^{-\mu} e^{\mu}
$$

From (9), because $N_{o}$ is a function of $N_{D}, D_{o}$, and $\mu$ and $\delta$ and $\mu$ are mutually coupled through $D_{o}$ and $D_{m}$, the parameters $N_{o}, \delta$ and $\mu$ in (4) cannot be treated as independent of each other.

Substituting (4) into (2), we have:

$$
\mathrm{Z}=N_{o} \frac{\Gamma(\mu+7)}{\delta^{\mu+7}}
$$

where $\Gamma(\mathrm{x})$ is the Gamma function. Note that the mean Doppler velocity $V_{D}$ of the VHF radar returns from precipitation particles with respect to the still air is defined as:

$$
V_{D}=\frac{\int V_{T}(D) N(D) D^{6} d D}{\int N(D) D^{6} d D} .
$$

Substituting (4) and (5) into (11) and performing the integration, we have:

$$
V_{D}=\frac{A \Gamma(B+\mu+7)}{\delta^{B} \Gamma(\mu+7)} .
$$

Eliminating $\delta$ in (12) in terms of (10), a power law relation between $V_{D}$ and $\mathrm{Z}$ is derived as follows (Ulbrich and Chilson 1994):

$$
V_{D}=\frac{A \Gamma(B+\mu+7)}{N_{0}^{B /(\mu+7)}[\Gamma(\mu+7)]^{(B+\mu+7) /(\mu+7)}} Z^{B /(\mu+7)} .
$$

Because the VHF backscatter of precipitation is related to reflectivity in accordance with (1), the relation between mean Doppler velocity and the VHF backscatter can be formulated below:

$$
V_{D}=\alpha P^{B},
$$

where $P=P_{r} \times r^{2}$, and

$$
\alpha=\frac{A \Gamma(B+\mu+7)}{\Gamma(\mu+7)^{(1+\beta)}\left(N_{0}|K|^{2} C\right)^{\beta}}
$$




$$
\beta=\frac{B}{\mu+7}
$$

From (14), (15), and (16), a positive correlation between $V_{D}$ and $P$ is expected because $A, \mu$, and $B$ are always positive. It is noteworthy that the values of $\alpha$ and $\beta$ can be estimated by best fitting (14) to $V_{D}$ and $P$, which can be both obtained from the Doppler spectrum of the VHF radar echoes from precipitation.

From (15) and (16), we note that the mathematical relation between $\alpha$ and $\beta$ is very complicated, in which the precipitation-related parameters $N_{o}, \mu, A$ and $B$ are coupled together and cannot be easily separated from each other. As a result, although expressions (15) and (16) contain the information on the drop size distribution and fallspeed-diameter relation of the precipitation particles, they cannot be readily used to estimate separately the parameters $N_{o}, A, \mu$, and $B$. Therefore, developing a more simplified relation between $\alpha$ and $\beta$ is required such that the separation of $N_{o}$ and $\mu$ from $A$ and $B$ in (15) and (16) is possible. For this reason, we expand the Gamma function in (15), after taking the logarithm, in a form of asymptotical approximation as follows (Bender and Orszag 1978):

$$
\ln \Gamma(x)=\ln \left(\sqrt{2 \pi} x^{x-\frac{1}{2}} e^{-x}\right)+\frac{1}{12 x}-\frac{1}{360 x^{2}}+\ldots
$$

where $x$ is equal to $\mu+7$ or $B+\mu+7$. Note that, in light of the fact that the order of magnitude of $\mu+7$ is about 10 , which will be shown in next section, the second and higher terms in the right hand side of (17) can be reasonably neglected compared to the first term. Because of this and $0.5<B<0.7$, we have $\beta<<1$. From (17), after performing mathematical manipulation, we have:

$$
\ln \frac{\Gamma(B+\mu+7)}{[\Gamma(\mu+7)]^{(1+\beta)}} \cong \beta\left\lfloor\mu+7+\frac{\ln (\mu+7)}{2}-\sqrt{2 \pi}\right\rfloor,
$$

where the terms with $B^{2} /(\mu+7)$ are neglected and the approximation $\ln (1+\beta) \sim \beta$ is made in deriving (18). With expression (18), (15) can be approximated to:

$$
\alpha=A e^{\xi \beta}
$$

where

$$
\begin{aligned}
& \xi=M-N, \\
& M=\mu+7+\frac{\ln (\mu+7)}{2},
\end{aligned}
$$




$$
N=\sqrt{2 \pi}+\ln \left(N_{o}|K|^{2} C\right),
$$

Expression (19) clearly shows that the mathematical relation between $\alpha$ and $\beta$ can be described by a more simplified expression in a form of the exponential function, which verifies the empirical relation between $\alpha$ and $\beta$ found by Chu et al. (1999).

Superficially, one might think that the $A$ value in (5) can be directly obtained from (19) by letting $\xi \beta=0$, which is independent of the characteristics of the drop size distribution. However, later we will show that it is not the case. Figure 1 shows the comparison of $\alpha-\beta$ relations for analytical solution (solid curve) as shown in (15) and for exponential approximation (dashed curve) as shown in (19) - (22). The parameters employed for the calculation are that: $A=10 \mathrm{~m} \mathrm{~s}^{-1} \mathrm{~cm}^{-\mathrm{B}}$, $B=0.6, D_{o}=0.8 \mathrm{~mm}, N_{D}=5000 \mathrm{~m}^{-3} \mathrm{~mm}^{-1}, L=0.15,|K|^{2}=0.93, P t=70 \mathrm{~kW}, G_{t}=30 \mathrm{~dB}$, $G_{r}=29 \mathrm{~dB}, G=50 \mathrm{~dB}, \theta=\psi=0.129 \mathrm{rad}$ (corresponding to half-power-beam-width of $7.4^{\circ}$ ), $\lambda=5.77 \mathrm{~m}$ (corresponding to $52 \mathrm{MHz}$ ), and $\tau=2 \mu \mathrm{s}$. As shown, the exponential approximation is in excellent agreement with the analytical solution with the mean relative error less than 5.5\%. Inspecting Fig. 1 in more detail shows that for the case of $\beta=0$ the value of $\alpha$ is equal to 8.75 , which is clearly not equal to the given $A$ value 10 . This result can be explained below. We note from expressions (9), (16), (21), and (22) that the power $\xi \beta$ in (19) is a function of $\mu$, and its magnitude will approximate to a finite and non-zero value as $\mu$ approaches infinity (or equivalently $\beta$ is close to zero because $B$ in $\beta$ is a finite value within the range $0.5-0.7$ as mentioned above). In the condition of $\beta=0$, the magnitude of $\xi \beta$ can be computed by first substituting (9) into (22) and then combining with (21). After rearranging the mathematical expression and letting $\mu$ approach infinity, we have:

$$
\lim _{\mu \rightarrow \infty} \xi \beta=\lim _{\mu \rightarrow \infty} \frac{B\left[X+0.5 \ln (\mu+7)+\mu \ln D_{o}\right]}{\mu+7}=B \ln D_{o}
$$

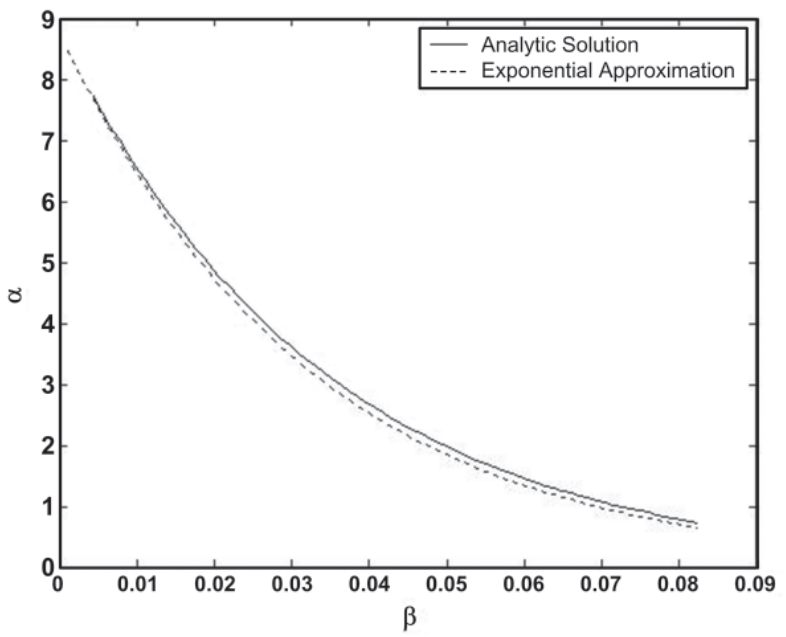

Fig. 1. Comparison of $\alpha-\beta$ relations between analytical solution and exponential approximation. For detailed description of the parameters employed for the calculation, see text. 
where $X\left[=7-\sqrt{2 \pi}-\ln \left(N_{D}|K|^{2} C\right)\right]$ in (23) is a parameter independent of $\mu$. Therefore, from (23), we note that the estimated $A$ value obtained in accordance with (19) by letting $\beta=0$ (or $\mu$ approximate to infinity) will be $A \exp \left(\mathrm{B} \ln D_{o}\right)$, rather than the real $A$ value itself. As a result, in order to obtain the real $A$ value from $\alpha-\beta$ relation as expressed in (19), the factor $\exp \left(-B \ln D_{o}\right.$ ) should be multiplied to the estimated $A$ value. Because the magnitude of $\exp \left(-B \ln D_{o}\right)$ is equal to 1.1428 for $B=0.6$ and $D_{o}=0.8$, and the magnitude of the estimated $A$ value is 8.75 as shown in Fig. 1, the true $A$ value $10(=8.75 \times 1.1428)$ is thus obtained. Therefore, the factor $\exp \left(-B \ln D_{o}\right)$ should be taken into account in the use of (19) to estimate the true $A$ value through the least squares fit.

The example presented in Fig. 1 shows that the correlation between $\alpha$ and $\beta$ is negative. Nevertheless, from (20) - (22) we note that the $\alpha-\beta$ relation is governed by the sign of $\xi$. Namely, the correlation will be positive if $M>N$ and negative if $M<N$. For Doppler radar with great transmitted power, high radar transfer gain, and large antenna gain, the negative correlation between $\alpha$ and $\beta$ will be expected because the magnitude of $C$ is usually so large that the value of $N$ is much greater than that of $M$, leading to negative sign of $\xi$. We will present experimental results in the next section to validate this assertion.

\section{EXPERIMENT SETUPS AND PRECIPITATION OBSERVATIONS}

\subsection{Disdrometer Observations}

Rain drop size distribution plays a critical role in developing a theoretical $V_{D}$ and $P$ relationship as presented in the above section. Different drop size distribution will give rise to different $V_{D}-P$ relations. A ground-based disdrometer deployed at the Chung-Li VHF radar site was employed to obtain information on rain drop size distribution. For detailed description of the characteristics of the disdrometer, refer to Schonhuber (1998) and Shih (2001). Figure 2 presents the time series of the surface rain fall rate recorded by the disdrometer. As indicated, the precipitations consist of a number of intense rain fall events which occurred intermittently with durations of about a few minutes and had peak intensities within a range from 4 to $50 \mathrm{~mm} \mathrm{hr}^{-1}$. Presumably, the clouds responsible for the precipitations, as shown in Fig. 2, are very likely to be the convective clouds which are characterized by active vertical air motions and intermittent rain fall events. We will present later the profiles of the backscatter from precipitation and vertical air velocity measured by the Chung-Li VHF radar to confirm this speculation. Figure 3 presents an example of the drop size distribution for the duration from 1923 to 1938 LT, in which asterisk points are the measured data and the solid curve is the best fit to the data in terms of the Gamma function as described in (4). As indicated in Fig. 3, the main body of the observed drop size distribution can be approximated very well to the Gamma distribution, irrespective of slight deviations of the observed data from the best fit curve at the small size end with drop diameter less than $0.5 \mathrm{~mm}$. In light of the fact that the Gamma distribution can nearly perfectly describe observed rain drop size distribution, it is adopted for the development of the theoretical expression of the $V_{D}-P$ relation in the above section. 


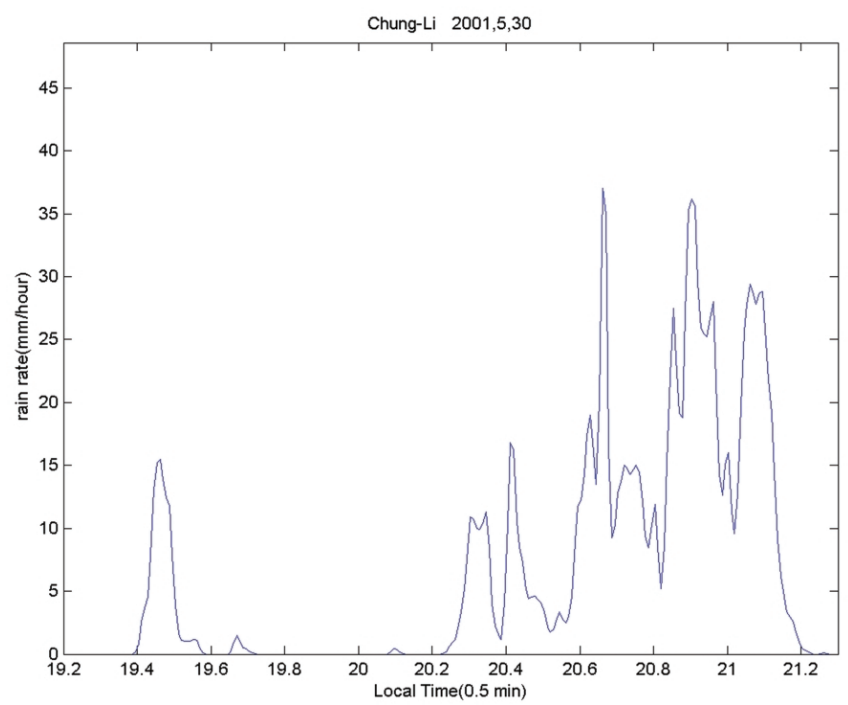

Fig. 2. Time series of surface rain rate recorded by optical rain gauge deployed on the campus of National Central University.

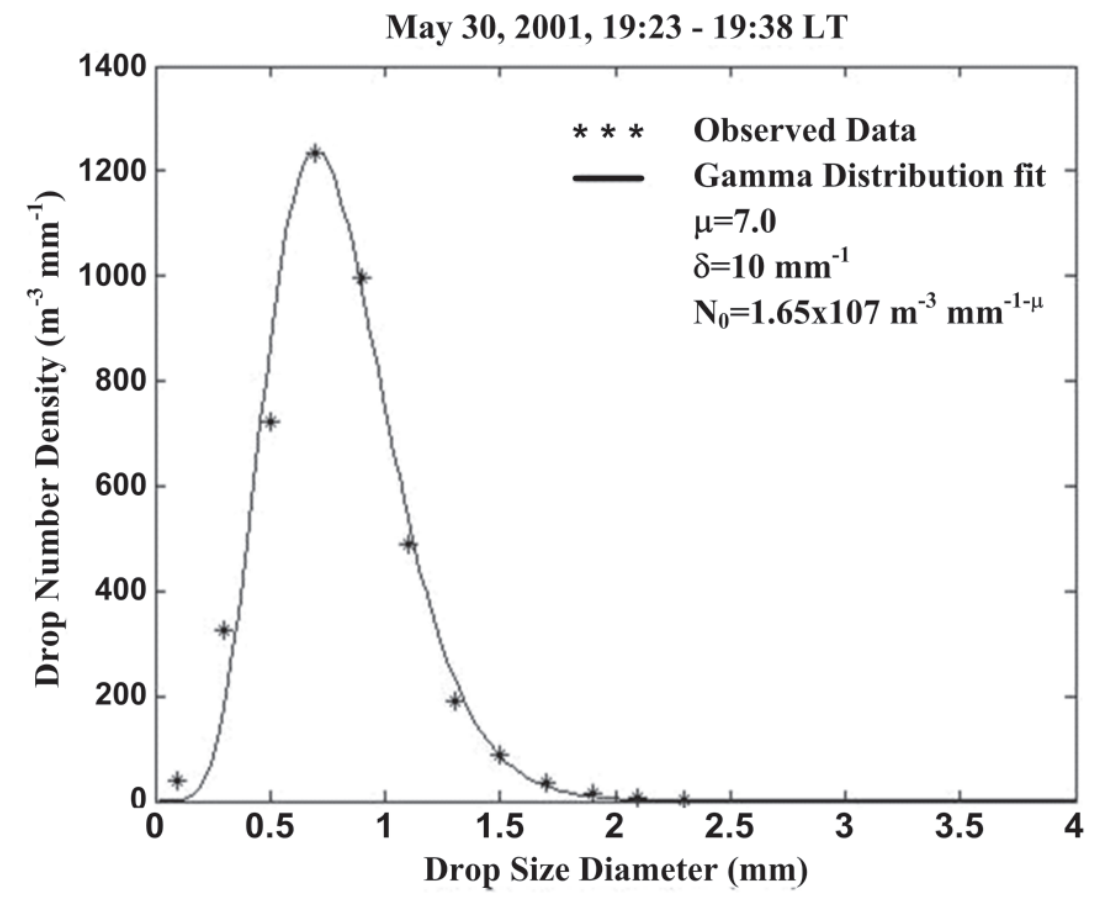

Fig. 3. An example of drop size distribution measured by a ground-based 2-dimensional discrometer, in which the solid curve is the best fit of the Gamma distribution to the data marked by discrete asterisks. The data were collected during the period 201949 to 203420 LT 30 May 2001. 


\subsection{Radar Observations}

The radar data employed for analysis were taken on May 30, 2001, by the Chung-Li VHF radar. The radar parameters were set as follows: nominal radar frequency was $52 \mathrm{MHz}$ (corresponding to wavelength of $5.77 \mathrm{~m}$ ), peak transmitted power was $70 \mathrm{~kW}$, interpulse period was $300 \mu \mathrm{s}$, pulse length was $2 \mu \mathrm{s}$, coherent integration number was 240 , delay time was $12 \mu \mathrm{s}$, forty range bins were set, and the antenna beam was steered vertically. The 128-point Fast Fourier Transform (FFT) algorithm was used to obtain a raw Doppler spectrum, and three raw spectra were incoherently integrated to obtain a resultant spectrum for the calculations of echo powers and Doppler velocities of the turbulent refractivity and precipitation particles. For detailed description of characteristics of the Chung-Li VHF radar for precipitation measurement, refer to Chu et al. (1999).

Figure 4 shows a typical height variation of the Doppler spectra observed by a vertically pointed radar beam of the Chung-Li radar, in which precipitation echoes (corresponding to the

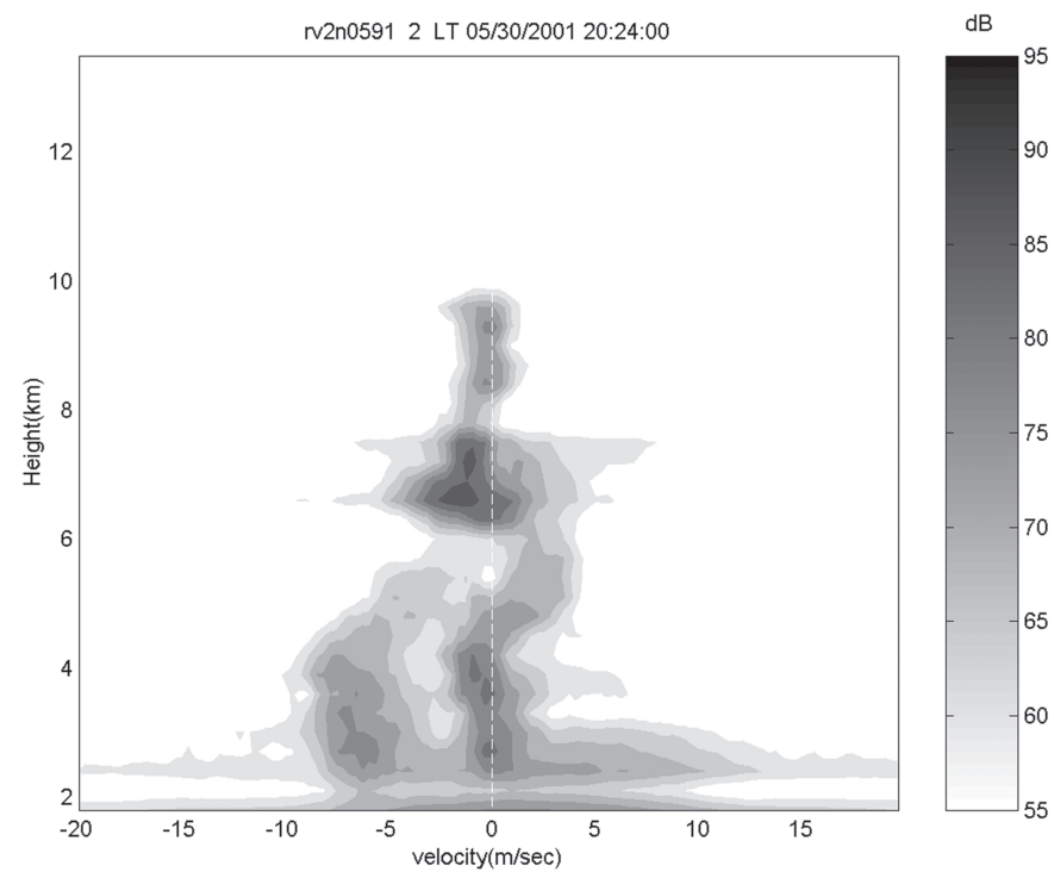

Fig. 4. An example of typical height variation of the Doppler spectra for the VHF radar echoes in precipitating environment, in which the precipitation echoes (corresponding to the spectral components with negative Doppler velocities below around $5 \mathrm{~km}$ ) and turbulent refractivity echoes (spectral components with Doppler velocities varied around zero $\mathrm{m} \mathrm{s}^{-1}$ ) are clearly identified. 
spectral components with negative Doppler velocities below around $6 \mathrm{~km}$ ) and turbulent refractivity echoes (corresponding to spectral components with Doppler velocities varied between zero and positive values) are clearly identified. Because the vertical air velocity can be estimated directly from the Doppler spectrum of turbulent refractivity, true terminal velocity of the precipitation particle can thus be estimated by removing the contribution of vertical air velocity from the observed Doppler velocity of the precipitation echoes. As indicated in Fig. 4, vertical air velocity as large as $3 \mathrm{~m} \mathrm{~s}^{-1}$ or more in the height range $5-6 \mathrm{~km}$ is seen. Accompanying the intense updraft, striking height variations in fall velocities of rain drops are observed. The fall velocities of rain drops vary from $2.8 \mathrm{~m} \mathrm{~s}^{-1}$ at about $5.5 \mathrm{~km}$ to $6.2 \mathrm{~m} \mathrm{~s}^{-1}$ at $2.4 \mathrm{~km}$. Note that, from the temperature profile measured by Pan-Chiao radiosonde station located about 25 $\mathrm{km}$ northeast of the Chung-Li radar site, the level of $0^{\circ} \mathrm{C}$ isotherm is located at about $6 \mathrm{~km}$. Because of the difference in the magnitudes of the complex refractive index, the backscattered powers from liquid rain drops will be greater than those from dry ice particles by $6.74 \mathrm{~dB}$ (Battan 1973). Therefore, the enormously strong echo powers with small negative Doppler velocity (toward radar) less than $2 \mathrm{~m} \mathrm{~s}^{-1}$ that occurred in the height range $6-8 \mathrm{~km}$ above the melting level with peak intensity much larger than that just below the melting level (more than $30 \mathrm{~dB}$ ) are unlikely to be originated solely from dry ice particles. Presumably, these intense radar echoes, occurring above the melting level, are probably associated with the scattering of supercooled water droplets accompanied by intense updraft which supplies sufficient water vapor for the formation of the supercooled droplets. However, more experiments are needed to verify this speculation, such as a radar experiment operated at multiple frequencies, airplane in situ measurement, multi-band microwave radiometer observations, and so on.

Figure 5 presents the height time variation of the vertical air velocity for the precipitation events shown in Fig. 2. As indicated, a number of intense updrafts with vertical velocity as large as greater than $4 \mathrm{~m} \mathrm{~s}^{-1}$ occurred intermittently in the height range from $2.4 \mathrm{~km}$ to above $6 \mathrm{~km}$, and each updraft event is characterized by short duration (lasting only a few minutes) and large height coverage (extending from $1 \mathrm{~km}$ to more than $2.5 \mathrm{~km}$ ). These features clearly indicate that the clouds responsible for the precipitations are convective clouds with active air motions that can reach an altitude above $7 \mathrm{~km}$. Figure 6 shows the height time intensity plot of VHF backscatter from precipitation particles. Comparing Fig. 6 with Fig. 5 reveals that the occurrence of precipitation backscatter coincided generally with that of updraft. In addition, inspecting Fig. 6 shows that no bright band structure was found around the melting-level height. Obviously, the precipitation process responsible for the rainfall event can be attributed to the warm precipitation process, namely, the condensation of water vapor carried by an intense updraft and the subsequent coalescence of small droplets during their fall. This is the primary mechanism responsible for the formation of the rain drops below the melting level.

Figure 7 presents a contour of the terminal velocity of the rain drops. As shown, terminal velocities as large as $9 \mathrm{~m} \mathrm{~s}^{-1}$ or more were observed during the periods when intense updrafts occurred. As shown, large precipitation particles with large terminal velocities might appear not only at lower height, but also in the upper altitude. Comparing Figs. 5 and 7 demonstrates that the occurrence of large rain drops in the upper level is coincident with the existence of intense updraft. This feature clearly indicates that intense updraft is a crucial factor in the formation of large rain drops at upper altitude. 


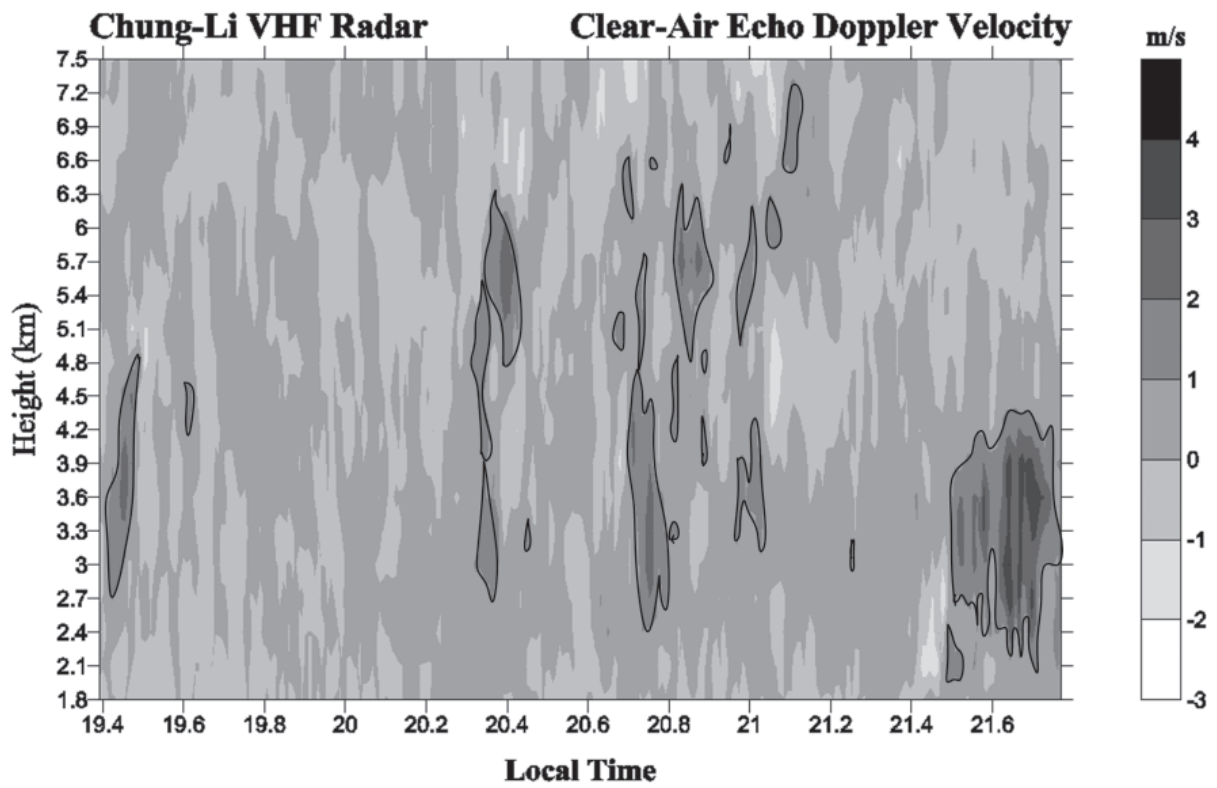

Fig. 5. Height time variation of observed vertical air velocity.

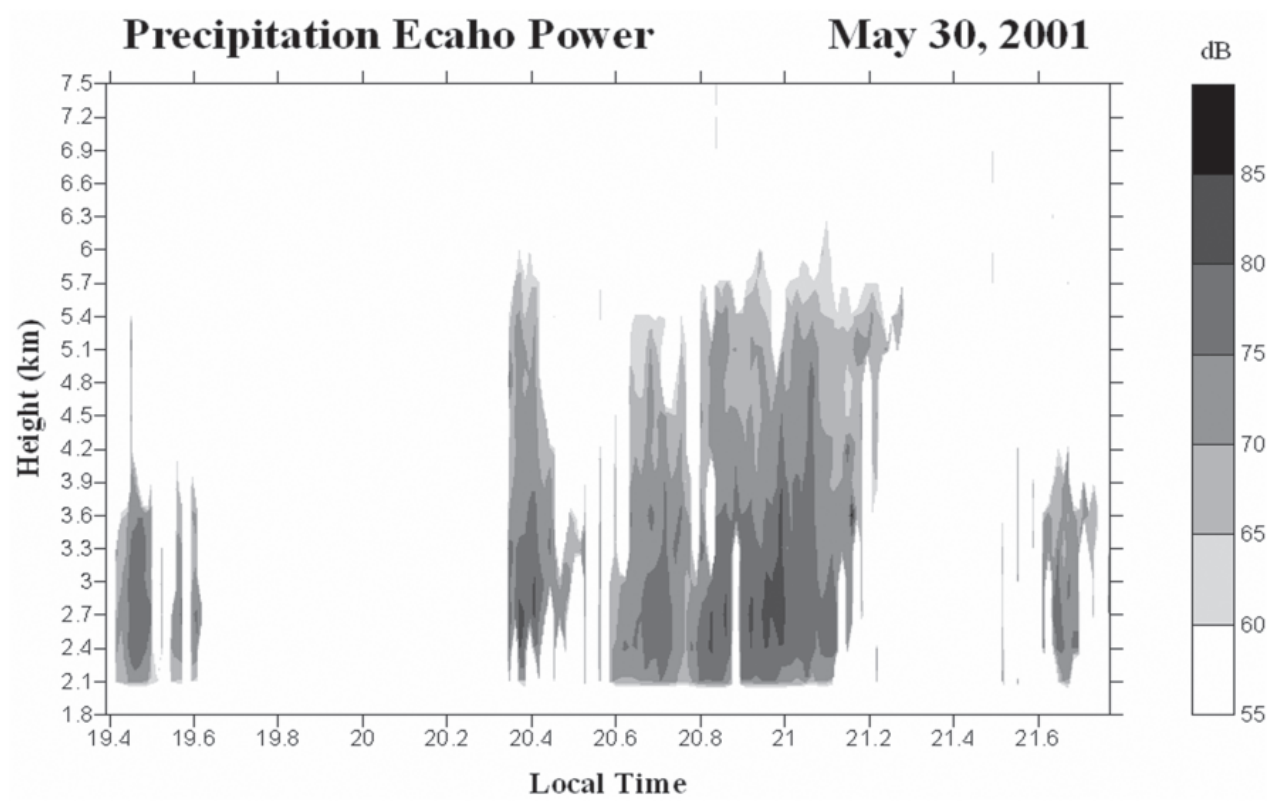

Fig. 6. Height-time-intensity contour plot of the precipitation backscatter. 


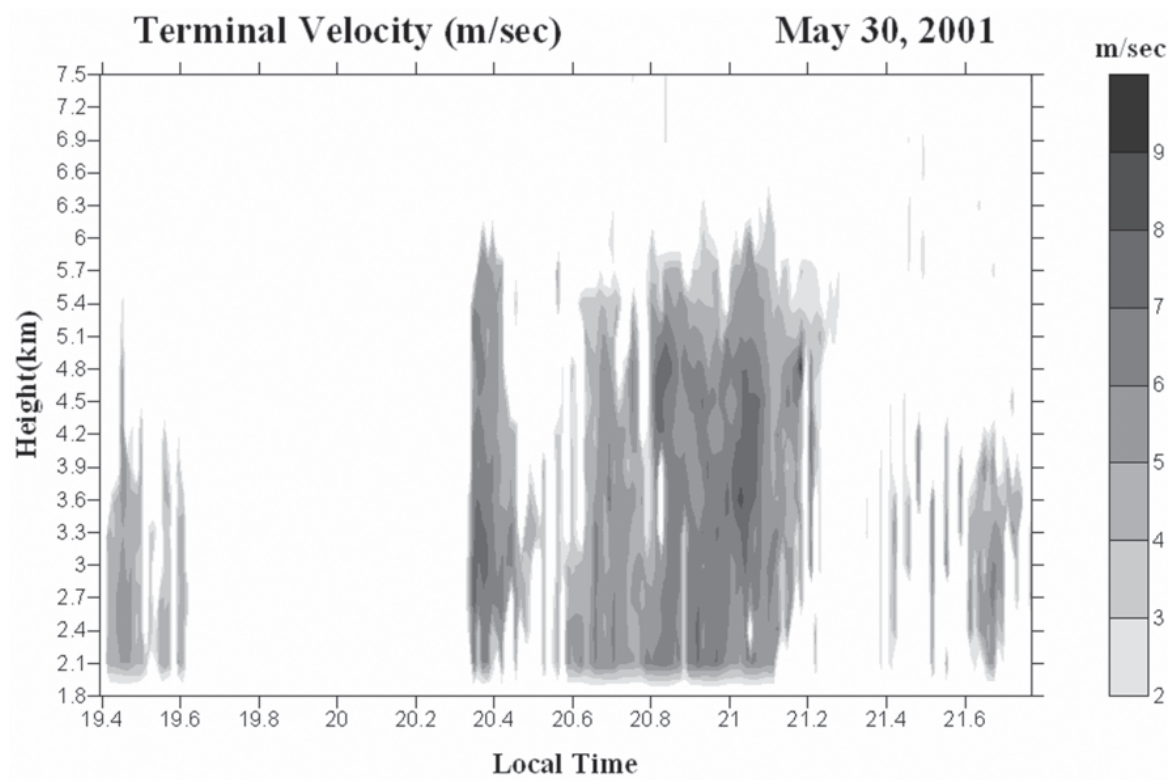

Fig. 7. Height-time-intensity contour plot of terminal velocity of the precipitation particles.

Figure 8 presents a scatter diagrams of range-corrected precipitation echo power $P$ (i.e., $P_{r} R^{2}, P_{r}$ is measured echo power and $R$ is the range) versus terminal velocity $V_{D}$ of precipitation in logarithmic scale at different altitudes, in which the symbols a and b marked above each panel correspond to $\alpha$ and $\beta$ in expression (14) estimated by best fitting (14) to the radar data and cc represents the correlation coefficient. Note that, in light of the fact that air density will affect the terminal velocity of a precipitation particle during its fall, a factor should be multiplied to the observed terminal velocity for the correction of the air density effect on the terminal velocity at different altitudes before carrying out the least squares fit. The correction factor employed in this study is $\left(\rho_{o} / \rho\right)^{0.4}$, where $\rho_{o}$ and $\rho$, are the air densities at the ground and at the level of radar observation, respectively (Foote and du Toil 1969; Atlas et al. 1973). As shown in Fig. 8, a positive correlation between $\mathrm{P}$ and $V_{D}$ is seen for the data above $2.1 \mathrm{~km}$, which is in agreement with the theoretical result as described in (14). Note that dramatic changes in $\alpha$ and $\beta$ values with height are seen. As shown in expressions (15) and (16), a number of parameters associated with precipitation particles determine the magnitudes of $\alpha$ and $\beta$, including $A, B, \mu$, and $N_{o}$. Therefore, the dramatic changes in $\alpha$ and $\beta$ may be indicative of the changes in these parameters with height. Once the values of $\alpha$ and $\beta$ are obtained, one might expect that their relation can be approximated to an exponential function described by (19) and the height-averaged coefficient $A$ and power $\xi$ in (19) can thus be estimated through leastsquares fit. Figure 9 shows the best fit of expression (19) to observed data, in which the open circles are the data taken from Fig. 8. It is clear that the fitted curve is in excellent agreement with the data, validating the exponential approximation to $\alpha-\beta$ relation. 

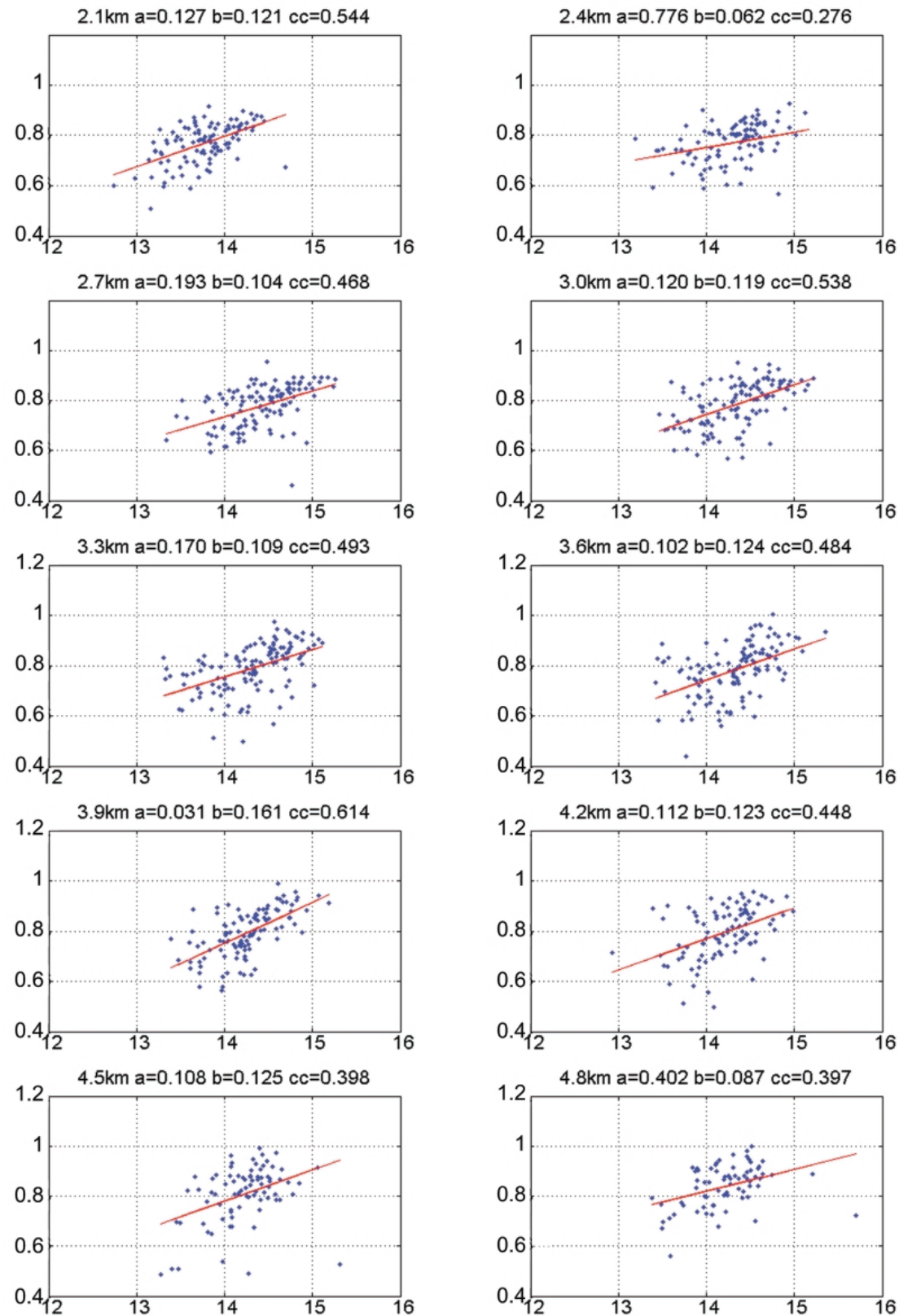

Fig. 8. Scatter diagrams of range-corrected precipitation echo power (i.e., $P_{r} r^{2}$, $P_{r}$ is measured echo power and $r$ is the range) versus terminal velocity $V_{D}$ of precipitation at different altitudes, in which the symbols a and $\mathrm{b}$ above each panel are, respectively, $\alpha$ and $\beta$ in expression (15) estimated through least-squares fit and cc represents the correlation coefficient of the data. The period of the data was from 2035 to 2110 LT. 


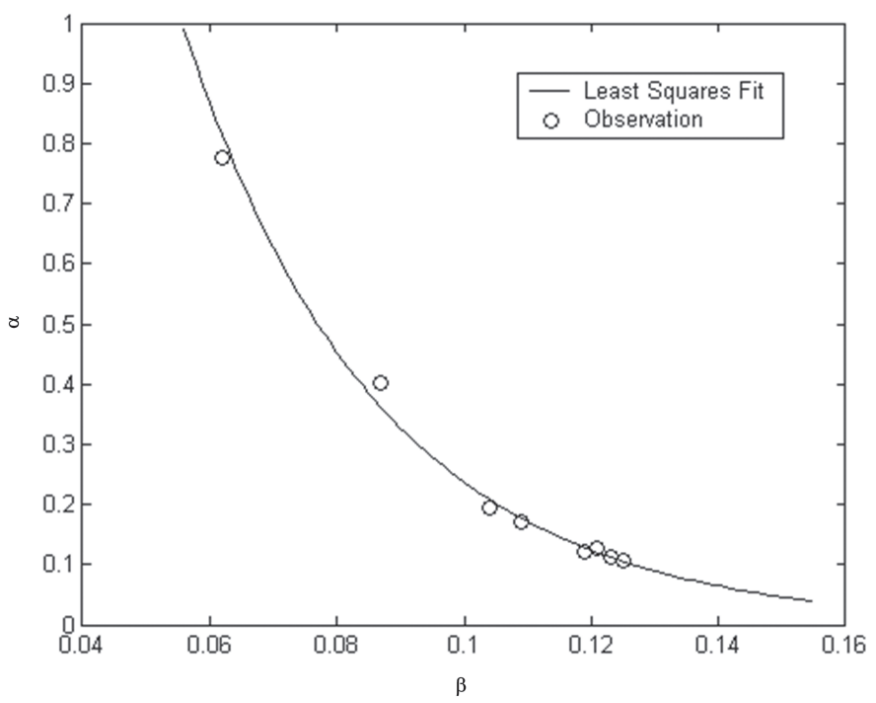

Fig. 9. Best fit of expression $\alpha=A \exp (-\xi \beta)$ to the estimated $\alpha$ and $\beta$, where the data are taken from Fig. 8.

\section{DISCUSSION}

In section 2, assuming the Gamma distribution of the rain drop size and power law relation between terminal velocity and the size of precipitation particle, we find that the mathematical relation between $\alpha$ and $\beta$ for the expression (15) can be perfectly approximated to a very simple exponential function as described in (19). Examining (19) in more detail indicates that, except for the radar parameter $|K|^{2} C$, the magnitude of $\xi$ is the function of $\mu$ and $N_{o}$. In consequence of this connection, $\xi$ has the potential to deduce the information on the parameters $N_{o}$ and $\mu$ of drop size distribution. From expressions (20) - (22), the absolute value of $\xi$ is related to $N_{o}$ and $\mu$ as follows:

$$
|\xi|=\ln \left(\frac{N_{o}}{e^{\mu} \sqrt{\mu+7}}\right)+\chi
$$

where $\chi$ is a constant as a function of $|K|$ and $C$ and bears no relation on the rain drop distribution. Figure 10 is the scatter diagram of $|\xi|$ versus $\ln \left(N_{o} /\left[\exp (\mu)(\mu+7)^{0.5}\right]\right)$, where the former are estimated from the VHF radar returns generated from the precipitations above $2.0 \mathrm{~km}$ and the latter are obtained from the rain drop distributions measured by the groundbased disdrometer. As shown in Fig. 10, a positive correlation between radar-estimated $\xi$ and disdrometer-measured $\ln \left(N_{o} /\left[\exp (\mu)(\mu+7)^{0.5}\right]\right)$ is seen, validating the theoretical expressions (19) - (22). In light of the close dependence of $\xi$ on drop size distribution, it seems to suggest that the general property of the drop size distributions in the air is approximate to that on the ground. Nevertheless, inevitable differences between the parameters $N_{o}$ and $\mu$ in the air and on the ground are expected owing to the height variations in dynamic behavior and the thermal structure of the air and spatially inhomogeneous distribution of the precipitation particles in the ambient atmosphere, causing the noticeable scatters of data points in Fig. 10. 


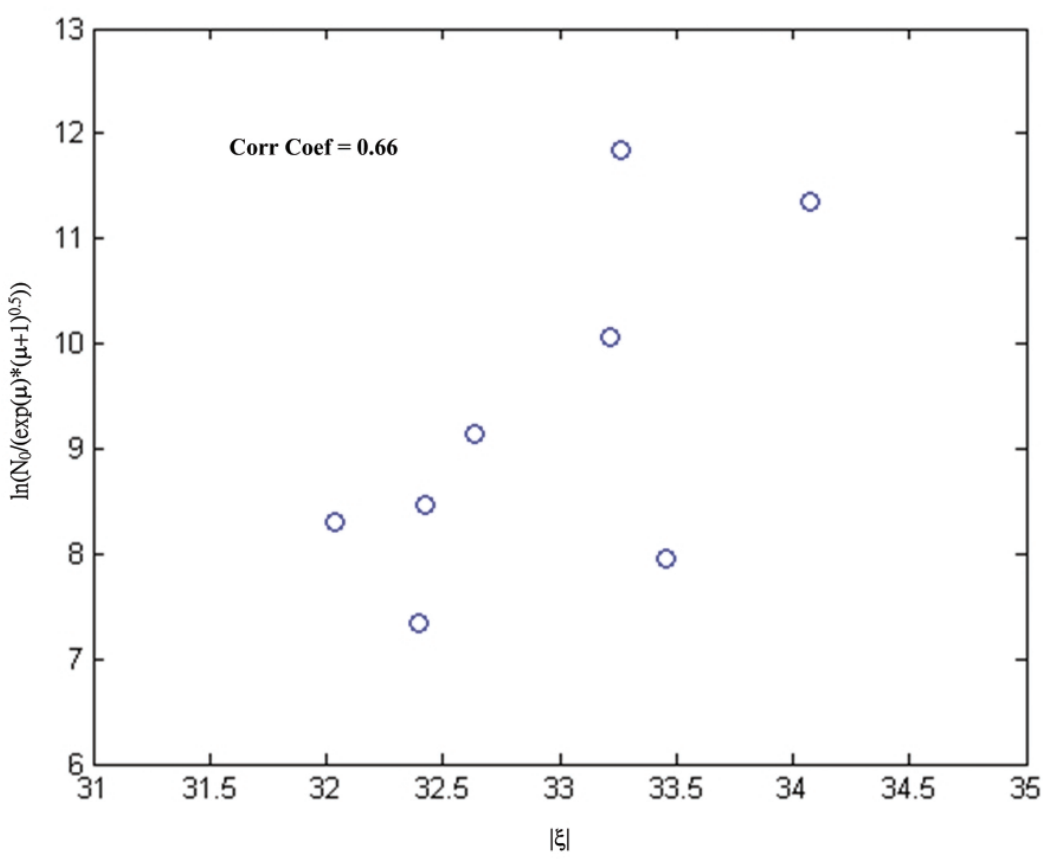

Fig. 10. Scatter diagram of the absolute values of $\xi$ versus the magnitudes of $\ln \left(N_{o} /\left[\exp (\mu)(\mu+7)^{0.5}\right]\right)$, where the former are estimated from the VHF precipitation backscatter above $2.0 \mathrm{~km}$ and the latter are obtained from the parameters $N_{o}$ and $\mu$ of the rain drop distributions measured by the ground-based disdrometer.

It is noteworthy that the precipitation event that we study in this research resulted from convective clouds with active air motions. Figures 2 and 6 demonstrate that appreciable changes in precipitation intensities with time are seen not only in the time series of the rainfall rate on the ground, but also in the radar backscatter in the air. However, the analysis of the cross correlation between radar backscatter and disdrometer-measured rainfall rate does not show organized and salient time delays between them. Namely, the systematic time delays are not seen in the profiles of the VHF radar backscatter, implying that the precipitation intensities at the different heights in the entire air column over the radar site are comparable and not changed significantly. As a result, it is expected that the rainfall rate recorded by the disdrometer on the surface will be approximate to that deduced from the precipitation echoes in the air. The definition of the rain fall rate $\mathrm{R}$ is given below:

$$
R=\frac{\pi}{6} \int N(D) D^{3} V(D) d D
$$


Substituting (4) and (5) into (25), we have:

$$
R=\frac{\pi A N_{o} \Gamma(B+\mu+4)}{6 \delta^{B+\mu+4}} .
$$

Note that the units of $A, N_{o}$, and $\delta$ are, respectively, $\mathrm{m} \mathrm{s}^{-1} \mathrm{~cm}^{-\mathrm{B}}, \mathrm{m}^{-3} \mathrm{~mm}^{-1-\mu}$, and $\mathrm{mm}^{-1}$ if $N(D)$ is in unit of $\mathrm{m}^{-3} \mathrm{~mm}^{-1}$. Therefore, the right hand side of (26) should be multiplied by a factor of $10^{-\mathrm{B}-9}$ such that $R$ is in unit of $\mathrm{m} \mathrm{s}^{-1}$. To convert the more commonly used unit of millimeter per hour, we need to multiply (26) by another factor $3.6 \times 10^{6}$. Figure 11 displays the scatter diagram of rainfall rates measured by the ground-based disdrometer versus those estimated in accordance with (26), in which the parameters $N_{o}, \mu$, and $\delta$ are extracted from the disdrometermeasured rain drop size distribution and $\mathrm{A}$ is estimated from the precipitation radar returns by means of (19). As indicated, the main body of the data points generally distribute along the line with slope of 1 , supporting the applicability of the exponential approximation to $\alpha-\beta$ relation for the rainfall rate estimated.

Chu et al. (1999) assumed the exponential drop size distribution and power law fall speeddiameter relation of precipitation particle to investigate $V_{D}-P$ relation. Under these assumptions, Chu et al. (1999) and Su et al. (2004) derived the theoretical $\alpha-\beta$ relation as follows:

$$
\alpha=\frac{A \Gamma(B+7)}{\Gamma(7)\left[\Gamma(7) N_{o}|K|^{2} C\right]^{\beta}},
$$

where $\beta=B / 7$. As a matter of fact, (27) can be achieved directly from (15) by giving $\mu=0$, which is corresponding to the special case of exponential distribution. Chu et al. (1999) also analyzed the characteristics of (27) and empirically found that (27) can be perfectly approximated to the same form of the exponential function as (19). Although Chu et al. (1999) did not derive the analytical expression of $\xi$ as a function of $A, B, N_{o},|K|^{2}$, and $C$, this work was completed by Su et al. (2004). If we substitute $\mu=0$ into (20) - (22), the corresponding expression of $\xi$ for the exponential drop size distribution will be obtained as follows:

$$
\xi=7+0.5 \ln 7-\sqrt{2 \pi}-\ln \left(N_{o}|K|^{2} C\right) .
$$

Alternatively, the analytical expression of $\xi$ for exponential drop size distribution can be obtained by substituting the asymptotic approximation of the Gamma function as shown in (17) into (27). After performing the same mathematical manipulation as Gamma drop size distribution, we obtain:

$$
\xi=7 \ln 7-0.5-\ln (\Gamma(7))-\ln \left(N_{o}|K|^{2} C\right) .
$$

Obviously, the magnitudes of $\xi$ for (28) and (29) are different, and computation shows that the latter is greater than the former by 1.07 . This difference is believed to arise from the math- 


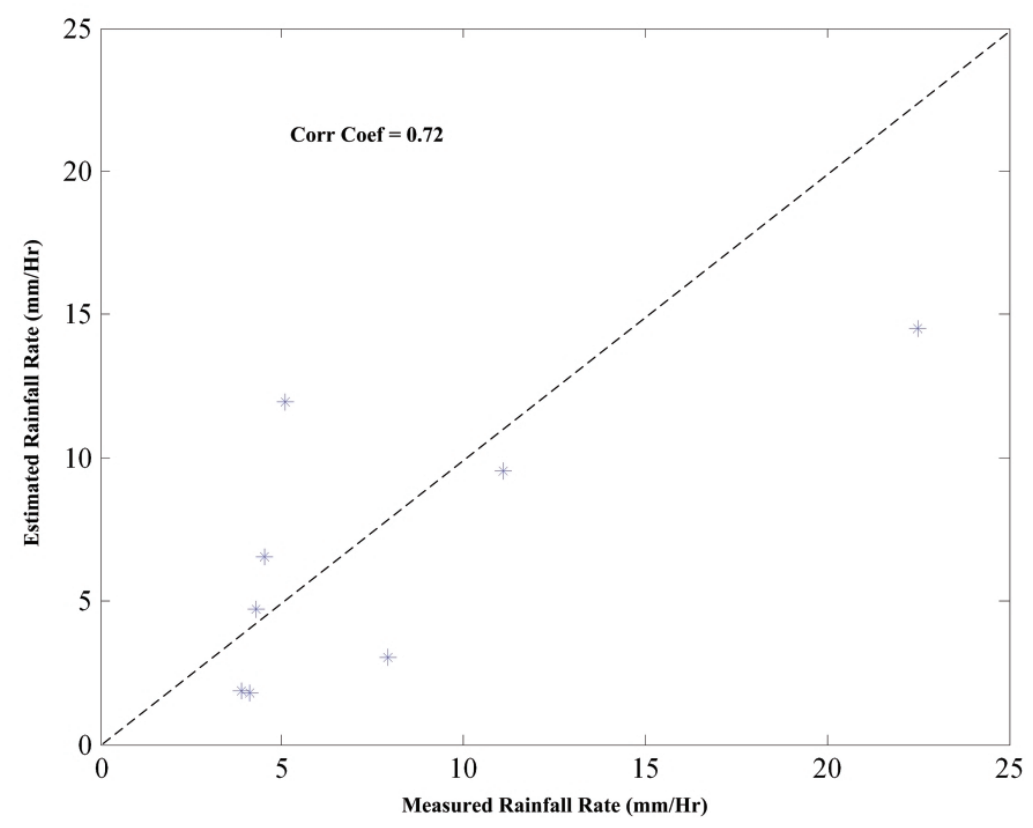

Fig. 11. Comparison of estimated rain fall rate in accordance with (26) and disdrometer-measured rain fall rate. The values of the parameters $N_{o}, A$, $\mu$, and $\delta$ employed for the estimation of the rainfall rate are listed in the Table 1.

Table 1. Precipitation parameters estimated from disdrometer-measured rain drop distributions and VHF radar echoes. The meanings of the parameters, see text.

\begin{tabular}{cccccc}
\hline $\begin{array}{c}\text { Period } \\
(\mathrm{LT})\end{array}$ & $\mu$ & $\begin{array}{c}\delta \\
\left(\mathrm{mm}^{-1}\right)\end{array}$ & $\begin{array}{c}\mathrm{N}_{\mathrm{o}} \\
\left(\mathrm{m}^{-3} \mathrm{~mm}^{-1-\mu}\right)\end{array}$ & $\xi$ & $\begin{array}{c}\mathrm{A} \\
\left(\mathrm{m} \mathrm{s}^{-1} \mathrm{~cm}^{-\mathrm{B}}\right)\end{array}$ \\
\hline $19: 23-19: 38$ & 07.0 & 10.0 & $1.65 \mathrm{e}+07$ & -32.174 & 6.23 \\
$20: 19-20: 34$ & 08.5 & 13.5 & $4.50 \mathrm{e}+08$ & -33.215 & 8.82 \\
$20: 34-20: 42$ & 11.6 & 16.4 & $4.00 \mathrm{e}+10$ & -33.1 & 7.28 \\
$20: 42-20: 50$ & 13.1 & 18.8 & $3.45 \mathrm{e}+11$ & -33.26 & 6.77 \\
$20: 50-20: 57$ & 05.5 & 08.0 & $8.00 \mathrm{e}+06$ & -34.07 & 9.20 \\
$20: 57-21: 05$ & 04.7 & 06.4 & $1.08 \mathrm{e}+06$ & -32.03 & 7.61 \\
$21: 05-21: 13$ & 04.3 & 06.4 & $3.85 \mathrm{e}+05$ & -32.717 & 7.68 \\
$21: 36-21: 46$ & 04.8 & 06.5 & $2.00 \mathrm{e}+06$ & -32.52 & 7.01 \\
\hline
\end{tabular}


ematical simplification in the development of (20) - (22) for the case of Gamma distribution. Therefore, a factor of 1.07 should be compensated to $\xi$ in (28) if it is used to deduce the value of $\xi$ for the exponential drop size distribution.

\section{CONCLUSION}

On the basis of Gamma drop size distribution and power law approximation to the fall speed $V_{T}$ and diameter $D$ of a precipitation particle, we show that the relation between mean terminal velocity $V_{D}$ of the precipitation particle and VHF radar range-corrected backscatter $P$ from precipitation is in a form of the power law $V_{D}=\alpha P^{\beta}$. With the help of asymptotic approximation of the Gamma function, we theoretically analyze the relation between coefficient $\alpha$ and power $\beta$ and find that their relation can be perfectly described by the exponential expression $\alpha=A \exp (\xi \beta)$, where $A$ is the coefficient in the power law approximation to the $V_{T}-D$ relation and $\xi$ is a function of $\mu$ and $N_{o}$ associated with Gamma drop size distribution. In light of the fact that the coefficients $A$ and $\xi$ contain separate and different information on the precipitation particle, it is possible to deduce the drop size distribution and $V_{T}-D$ relation in the air in accordance with the analytic expression of the $\alpha-\beta$ relation, which are very difficult to obtain using other means. The experimental results obtained by the Chung-Li VHF radar verify the exponential approximation to the $\alpha-\beta$ relation and show a good correlation to the disdrometer-measured precipitation parameters.

Acknowledgement This work was partially supported by National Science Council of Republic of China (Taiwan) under grant NSC92-2111-M-008-001.

\section{REFERENCES}

Atlas, D., R. C. Srivastava, and R. S. Sekon, 1973: Doppler radar characteristics of precipitation at vertical incidence. Rev. Geophys. Space Phys., 11, 1-35.

Atlas, D., and C. W. Ulbrich, 1977: Path- and area-integrated rainfall measurements by microwave attenuation in the $1-3 \mathrm{~cm}$ band. J. Appl. Meteorol., 16, 1322-1331.

Battan, L. J., 1973: Radar observations of the atmosphere. University of Chicago Press, Chicago, $324 \mathrm{pp}$.

Bender, C. M., and S. Orszag, 1978: Advanced mathematical methods for scientists and engineers - Asymptotic Methods and Perturbation Theory, McGraw-Hill, Inc., New York.

Caylor, I. J., and A. J. Illingworth, 1987: Radar observations and modeling of warm rain initiation. Q.J. R. Meteorol. Soc., 113, 1171-1191.

Chilson, P. B., C. W. Ulbrich, M. F. Larsen, P. Perillat, and J. E. Keener, 1993: Observations of a tropical thunderstorm using a vertically pointing, dual-frequency, collinear beam Doppler radar. J. Atmos. Ocean. Technol., 10, 663-673.

Chu, Y. H., and L. P. Chian, 1991: Investigation of atmospheric precipitations by using ChungLi VHF Radar. Radio Sci., 26, 717-729. 
Chu, Y. H., and C. H. Lin, 1994: Severe depletion of turbulent echo power in association with precipitation observed by using Chung-Li VHF Radar. Radio Sci., 29, 1311-1320.

Chu, Y. H., T. Y. Chen, T. H. Lin, 1997: An examination of the wind-driven on the drift of precipitation particles using the Chung-Li VHF Radar. Radio Sci., 32, 957-966.

Chu, Y. H., S. P. Shih, C. L. Su, K. L. Lee, T. H. Lin, and W. C. Liang, 1999: A study on the relation between terminal velocity and VHF backscatter from precipitation particles using the Chung-Li VHF Radar. J. Appl. Meteorol., 38, 1720-1728.

Foote, N. H., and P. S. du Toit, 1969: Terminal velocity of raindrop aloft. J. Appl. Meteorol., 8, 585-591.

Gunn, R., and G. D. Kinzer, 1949: The terminal velocity of fall for water droplets in stagnant air. J. Meteorol., 6, 243-248.

Hauser, D., and P. Amayenc, 1981: A new method for deducing hydrometeor-size distributions and vertical air motions from Doppler radar measurements at vertical incidence. J. Appl. Meteorol., 43, 547-555.

Larsen, M. F., and J. Rottger, 1987: Observation of thunderstorm reflectivities and doppler velocities measured at VHF and UHF. J. Atmos. Ocean. Technol., 4, 151-159.

Leary, C. A., and R. A. House, Jr., 1979: Melting and evaporation of hydrometeors in precipitation from the anvil clouds of deep tropical convection. J. Atmos. Sci., 36, 669-679.

Rao, T. N., D. N. Rao, and S. Raghavan, 1999: Tropical precipitating systems observed with Indian MST radar. Radio Sci., 34, 1125-1139.

Reddy, K., S. P. Shih, and Y. H. Chu, 2002: A study of precipitating cloud system using Chung-Li VHF radar. Radio Sci., 37, doi: 10.1029/2000RS002544.

Roettger, J., C. H. Liu, C. J. Pan, and S. Y. Su, 1995: Characteristics of lightning echoes observed with VHF ST Radar. Radio Sci., 30, 1085-1097.

Schonhuber, D. M., 1998: About interaction of precipitation and electromagnetic waves. $\mathrm{Ph}$. D Thesis, Technical University-Graz, Austria, $181 \mathrm{pp}$.

Sekhon, R. S., and R. C. Srivastava, 1971: Doppler radar observations of drop-size distribution in a thundersotrm. J. Atmos. Sci., 28, 983-994.

Shih, S. P., 2001: Establishment of Ka band propagation channel model in Taiwan. Ph. D Thesis, National Central University, Taiwan, 203 pp. (in Chinese)

Spilhaus, A. E., 1948: Raindrop size, shape, and falling speed. J. Meteorol., 5, 108-110.

Su, C. L., Y. H. Chu, and C. Y. Chen, 2004: Analyzing the relationship between terminal velocity of raindrops and VHF backscatter from precipitation. Terr. Atmos. Ocean. Sci., 15, 629-645.

Ulbrich, C. W., and P. B. Chilson, 1994: Effects of variations in precipitation size distribution and fallspeed law parameters on relations between mean Doppler fallspeed and reflectivity factor. J. Atmos. Ocean. Technol., 11, 1656-1663.

Wakasugi, K., A. Mizutani, M. Masaru, S. Fukao, and S. Kato, 1986: A direct method for deriving drop-size distribution and vertical air velocities from VHF doppler radar spectra. J. Atmos. Ocean. Technol., 3, 623-629.

Su, C. L., and Y. H. Chu, 2007: Analysis of terminal velocity and VHF backscatter of precipitation particles using Chung-Li VHF radar combined with ground-based disdrometer. Terr. Atmos. Ocean. Sci., 18, 97-116. 